\title{
Short time vibrational dynamics of excited metal
}

\section{nanoparticles}

\author{
Florian Dufey*and Sighart F. Fischer ${ }^{\dagger}$ \\ Institut für Theoretische Physik T38, Technische Universität München, \\ D-85748 Garching, Germany
}

January 14, 2007

\begin{abstract}
The vibrational dynamics of metal nanoparticles following excitation with fs-laser-pulses is considered. The induced electronic and lattice pressure contributions cause excitation of breathing modes in spherical particles. Special attention is paid to the role of the excitation of overtones, which affect the short-time dynamics substantially. For instance, the expansion of clusters increases linearly with time. The overtones also lead to shifts of the position of the minima and maxima of the cluster radius as a function of time. They are of comparable size as the shift due to inclusion of electron pressure effects in addition to the lattice pressure. Furthermore, the slope of the vibrations becomes discontinuous at certain time points. The proposed theoretical treatment is suited to describe the control of excitation of acoustic vibrations of nanoparticles.
\end{abstract}

*email: dufey@ph.tum.de

${ }^{\dagger}$ email: sighart.fischer@ph.tum.de 


\section{Introduction}

It has been found experimentally that an electronic excitation on the femtosecond time scale can induce vibrations of metallic nanoparticles $[1,2,3,4,5,6]$. Later, it became possible to distinguish different vibrational modes [7] and, very recently, to selectively excite and stop individual modes [8].

The first theoretical explanations of the vibrational dynamics proposed [2, $3,4,6]$ introduced certain approximations as they focused on the fundamental breathing modes. To obtain quantitative statements especially on the excitation of vibrational overtones, it is necessary to go beyond these approximations. This will lead to qualitatively different predictions about the excited vibrations especially on timescales comparable to the lifetime of the excited electronic states. The proposed theoretical treatment is well fitted to make predictions on how to control optically acoustic vibrations of nanoparticles as in the experiments reported in ref. [8].

The effect of the electronic excitation on the low frequency vibrations can be modeled by the time evolution of different pressure components $p$. This pressure components will not only depend on the energy density $E / V$ of the excitation but also on its character. The proportionality constant $\gamma$ is known as Grüneisen factor,

$$
p=-\frac{d E}{d V}=\gamma \frac{E}{V}
$$

The Grüneisen factor is known analytically for some special cases. If the energy $E$ is equal to the thermal energy of a hot ideal Fermi gas, $\gamma_{\text {Fermi }}=2 / 3$. We may also derive an expression for $\gamma$ when the energy is stored in plasma oscillations: The energy of the plasmon is proportional to the plasma frequency $\omega_{p}=\sqrt{n e^{2} / m \epsilon_{1}}$, where $n=N / V$ is the number density of electrons in the metal, $e$ and $m$ are the charge and mass, respectively, of the electrons and 
$\epsilon_{1}$ is the dielectric constant of the metal. We see that $E \propto V^{-1 / 2}$, so that $\gamma_{\text {plasmon }}=1 / 2$. Only when the excited states can be described by a temperature, as is the case with the hot Fermi gas or the hot lattice, we can write $E=\int C_{V} d T$. If the energy is thermal energy of a vibrating lattice, from the Debye approximation one infers $\gamma_{\text {Debye }}=3$. In the case of gold, one finds [4] experimentally $\gamma_{\text {lattice }}=2.7$.

In this letter, we shall derive detailed equations for the time dependence of the cluster's vibration once the pressure is known. Qualitatively, it is important that the Grüneisen factor is much lower for electronic excitations than for lattice excitations. Given that the primary excitation due to irradiation is of electronic nature, the Güneisen factor will increase strongly when the initial electronic excitation decays into thermal lattice excitation due to electron-phonon coupling.

As has been shown in refs. [3, 4], the electronic pressure, is important for a qualitatively correct description of the observed vibrational spectrum of the clusters as it sets in directly after the excitation, while the lattice pressure needs time to build up. However, the calculation of the induced vibration of the clusters in refs. $[2,3,4]$ is based on two assumptions which are too crude to allow for a detailed description of the observed overtone structure [7] or for an active control of the vibrations (cf. ref. [8]):

The first assumption consists in the use of the static bulk modulus $B$ or equivalently of the thermal expansion coefficient $\alpha$ in the calculation of the amplitude of the fundamental vibration mode. We shall show that the fundamental mode only contributes about $70 \%$ to the total thermal- or stress-induced static expansion, the other $30 \%$ being due to overtones. Hence, in this approximation the amplitude of the fundamental vibration mode will come out too large.

The second assumption consists in considering only the fundamental breath- 


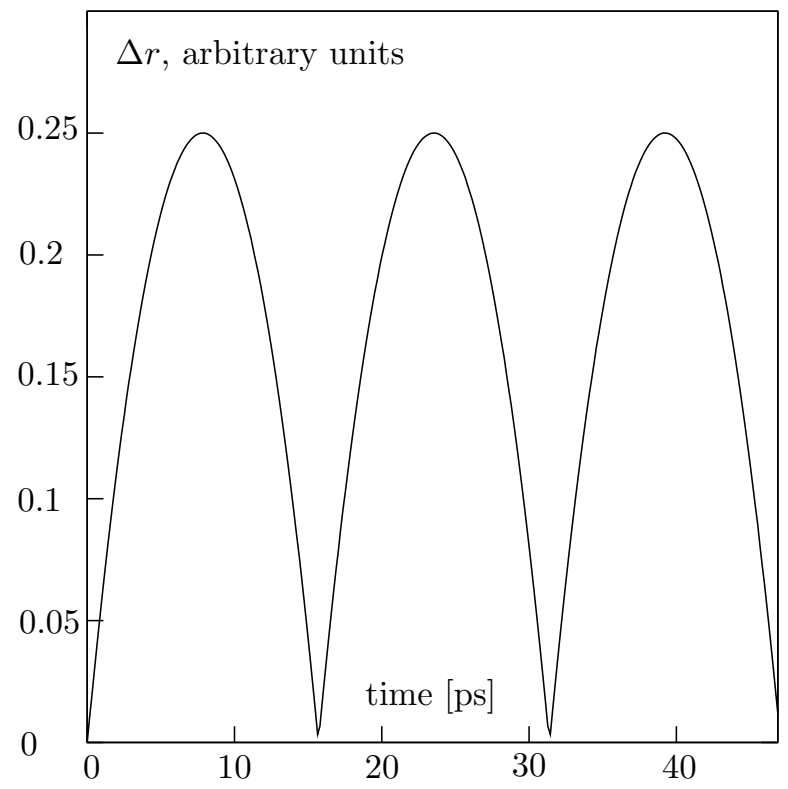

Figure 1: Radius change for a sudden change of pressure $p(t)=p_{0} \theta(t)$, cf. eq. (9). Due to the sudden excitation of overtones, the change is linear for short times. It may also be noted that the function has a discontinuous slope at points where $t$ is a multiple of $2 r_{0} / a_{l}$. The curve is a succession of parabolas. 
ing mode of the cluster. The generation of the electronic excitation by femtosecond laser pulses occurs on a timescale much shorter than the typical vibrational frequencies; hence we have to take into account the excitation of overtones. When these are included, the radius and volume of the cluster will increase linearly with time immediately after the laser excitation and not quadratically as one would predict when only the fundamental vibrational mode is taken into account.

While the first assumption changes the results only in a quantitative manner, the second assumption will change qualitatively the cluster's motion on a timescale where the energy of the cluster is still at least partly stored in electronic excitations which may have important consequences, e. g. on Förster type energy transfer between clusters.

\section{Calculation of the vibrational response to elec- tronic excitation}

We shall now derive the vibrational response of a spherical cluster to an increase of internal pressure. The cluster will be treated by continuum dynamics. To gain a feeling for the size of the quantities involved, we shall state the corresponding values for the case of gold, which is often used in experiments. We shall first study the free motion of the cluster and neglect damping [9] although inclusion of the latter presents no principal difficulties. In that case the equation of motion is $[10]$

$$
\rho \frac{\partial^{2} \mathbf{s}}{\partial t^{2}}=\nabla \cdot \sigma=(\mu+\lambda) \nabla \Theta+\mu \Delta \mathbf{s}
$$

Here, $\mathbf{s}$ is the displacement vector, $\mu$ and $\lambda$ are friction coefficients and $\sigma$ is the stress tensor with components $\sigma_{i k}=2 \mu \epsilon_{i k}+\lambda \delta_{i k} \Theta$. $\epsilon$ is the tensor of strain with components $\epsilon_{i j}=\frac{1}{2}\left(\partial_{i} s_{j}+\partial_{j} s_{i}\right)$. 


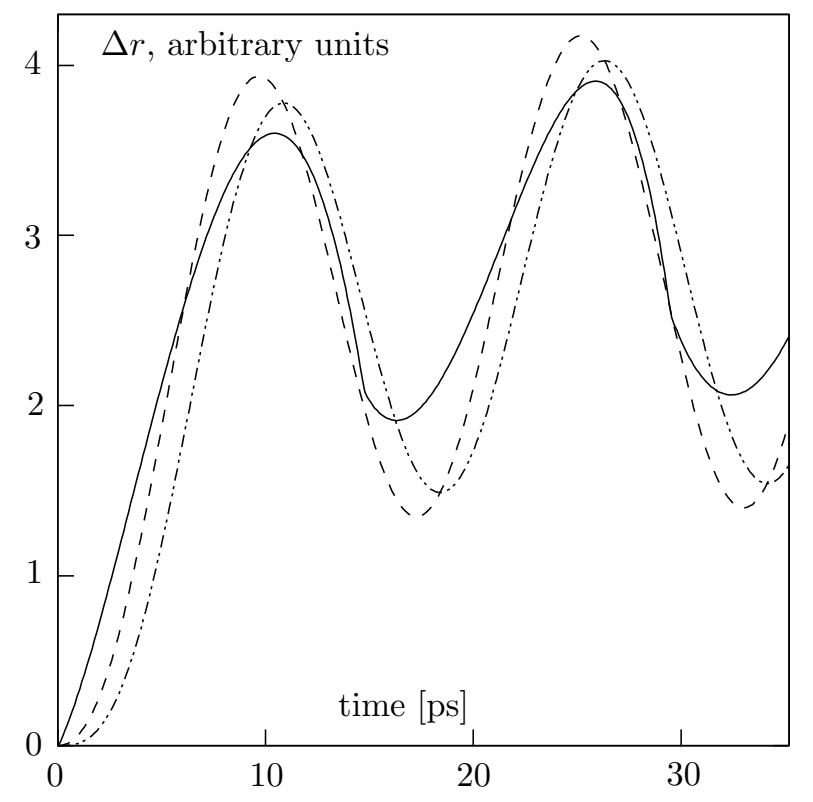

Figure 2: Vibration of a model gold cluster of radius $r_{0}=24.2 \mathrm{~nm}$. Full curve: Solution of eq. (7) with $p$ given by eq. (12). Dashed curve: Fundamental mode $(n=1)$ contribution to the exact solution, scaled due to the approximation $\frac{\left(K_{1} r_{0}\right)^{2}}{9} I_{2,1} \approx 1$. Dash-dotted curve: Fundamental mode contribution in the absence of electronic pressure $\left(\gamma_{\mathrm{Fermi}}=0\right)$. 
As $\Theta=\nabla \cdot \mathbf{s}$, upon forming the divergence, we get

$$
\frac{\partial^{2} \Theta}{\partial t^{2}}=a_{l}^{2} \nabla^{2} \Theta
$$

with the velocity of sound $a_{l}=\sqrt{(2 \mu+\lambda) / \rho}=3280 \mathrm{~m} / \mathrm{s}$ in the case of gold. We find the special solution $\Theta_{n}(r, t)=A_{0} \sin \left(\Omega_{n} t+\varphi_{n}\right) \sin \left(K_{n} r\right) /\left(k_{n} r\right)=$ $A\left(\Omega_{n} t\right) j_{0}\left(K_{n} r\right)$ with $\Omega_{n}=a_{l} K_{n}$ and $A\left(\Omega_{n} t\right)=A_{0} \sin \left(\Omega_{n} t+\varphi_{n}\right)$.

Taking into account the boundary condition $\sigma_{r r}=0$, which holds for clusters in vacuo, we obtain the well known equation $[11,12]$ for the wavenumbers $K_{n}$

$$
K_{n} r_{0} \cot K_{n} r_{0}=1-\frac{a_{l}^{2}}{4 a_{t}^{2}} K_{n}^{2} r_{0}^{2}
$$

where $a_{t}=\sqrt{\mu / \rho}$ is the transversal speed of sound. The number $n$ is greater or equal 1. In case of gold, $a_{t}=1190 \mathrm{~m} / \mathrm{s}$ and $\rho=19.3 \mathrm{~g} / \mathrm{cm}^{3}$ so that the exact result for the fundamental mode, which arises as a solution of eq. (3), is $K_{1} r_{0}=2.95$ for that material.

The electronic excitation will generate an additional pressure $p(r, t)$ whose precise value depends on the nature of the electronic excitation. Upon inclusion of this inhomogeneous term the stress tensor becomes $\sigma_{i k}=2 \mu \epsilon_{i k}+\lambda \delta_{i k} \Theta-$ $p(r) \delta_{i k}$. The pressure $p(r, t)$ is constant, $p(r, t)=p(t)$, in the range $0 \leq r \leq r_{0}$ and zero outside. We may expand this function into a series of the spherical Bessel functions $j_{0}\left(K_{n} r\right)$ with all possible values of $K_{n}$ that solve eq. (3)

$$
p(r, t)=p(t) \sum_{n=1}^{\infty} I_{1, n} j_{0}\left(K_{n} r\right)
$$

with coefficients

$$
I_{1, n}=\frac{\int_{0}^{r_{0}} r^{2} j_{0}\left(K_{n} r\right) d r}{\int_{0}^{r_{0}} r^{2} j_{0}^{2}\left(K_{n} r\right) d r} .
$$

The equation of motion decouples then into independent equations for each 
$\operatorname{mode} K_{n}$

$$
\frac{\partial^{2} \Theta_{n}}{\partial t^{2}}=-a_{l}^{2} K_{n}^{2} \Theta_{n}+\frac{p(t) I_{1, n}}{\rho a_{l}^{2}} a_{l}^{2} K_{n}^{2} j_{0}\left(K_{n} r\right)
$$

We see that the amplitude $A_{n}(t)$ will oscillate around the new equilibrium position $A_{\mathrm{eq}, n}=p I_{1, n} /\left(\rho a_{l}^{2}\right)$. Eq. (5) may be solved numerically for any time dependence of $p(t)$. The relative volume change of the whole sphere is equal to the average of $\Theta_{n}, \Delta V / V_{0}=\bar{\Theta}_{n}=\frac{3}{r_{0}^{3}} \int_{0}^{r_{0}} r^{2} \Theta_{n}(r) d r$. The volume change is related to the change of radius as $\Delta r_{n}=r_{0} \bar{\Theta}_{n} / 3$. Applying this averaging to eq. (5), we obtain an equation of motion for $\bar{\Theta}_{n}$,

$$
\frac{\partial^{2} \bar{\Theta}_{n}}{\partial t^{2}}=-\Omega_{n}^{2} \bar{\Theta}_{n}+\frac{p(t)}{\rho a_{l}^{2}} \Omega_{n}^{2} I_{2, n}
$$

with

$$
I_{2, n}=\frac{3}{\left(K_{n} r_{0}\right)^{3}} \frac{\left(\int_{0}^{K_{n} r_{0}} r^{2} j_{0}(r) d r\right)^{2}}{\int_{0}^{K_{n} r_{0}} r^{2} j_{0}^{2}(r) d r} .
$$

$M$ is the total mass of the cluster. In the case of gold $\left(K_{1} r_{0}=2.95\right) I_{2,1}=$ $0.708^{1}$.

Alternatively, we may write down an equation determining the time dependence of $\Delta r_{n}(t)$,

$$
\frac{\partial^{2} \Delta r_{n}}{\partial t^{2}}+\Omega_{n}^{2} \Delta r_{n}=\Omega_{n}^{2} r_{0} \frac{p(t)}{3 \rho a_{l}^{2}} I_{2, n}
$$

This has to be compared with the expression 2 in ref. [3]. The inhomogeneous term used in that article is in our nomenclature $4 \pi r_{0}^{2} p / M$. The approximation made is that the fundamental mode $(n=1)$ is the only mode that may lead to a volume change corresponds to setting $\frac{\left(K_{1} r_{0}\right)^{2}}{9} I_{2,1} \approx 1$. A similar approximation was invoked by Hartland [4]. Both Perner and Hartland only considered the fundamental vibrational mode. We shall now show that the overtones change

\footnotetext{
${ }^{1}$ For the homogeneous boundary condition $\Theta_{n}\left(r_{0}\right)=0$, which holds e. g. in a liquid, $K_{n} r_{0}=n \pi$. In that case, $I_{2, n}=6 /\left(n^{2} \pi^{2}\right)$. These boundary conditions give a qualitatively correct picture of the real behavior even in the case of isotropic solids.
} 
the picture qualitatively especially at short times.

\section{Examples}

We shall be interested in a time-interval where most of the excitation energy is still stored in the electronic subsystem, hence we assume that the pressure is equal to the electronic pressure immediately after the initial excitation pulse, whose width we assume to be infinitely small. Then we may approximate $p(t)=$ $p_{0} \theta(t)$. In that case eq. (7) has the analytical solution

$$
\bar{\Theta}_{n}=\bar{\Theta}_{\mathrm{eq}, n} \frac{1}{2}\left(1-\cos \Omega_{n} t\right),
$$

with $\bar{\Theta}_{\text {eq }, n}=\frac{p_{0}}{\rho a_{l}^{2}} I_{2, n}$. The total volume change is $\bar{\Theta}=\sum_{n} \bar{\Theta}_{n}$ for the short time after excitation, only the modes with high frequency $(n \gg 1)$ will have time to react. For these, we use the asymptotic values $I_{2, n} \sim \frac{6}{\pi^{2}} \frac{1}{n^{2}}$ and $\Omega_{n} \sim a_{l} \pi n / r_{0}$ to get for $t<2 r_{0} / a_{l}$ :

$$
\begin{aligned}
\bar{\Theta}(t) & \sim \frac{3 p_{0}}{\pi^{2} \rho a_{l}^{2}} \sum_{1}^{\infty} \frac{1}{n^{2}}\left(1-\cos a_{l} \pi n t / r_{0}\right) d n \\
& =\frac{3 p_{0}}{\rho a_{l}^{2}}\left(\frac{a_{l}}{2 r_{0}}\right)^{2} t\left(\frac{2 r_{0}}{a_{l}}-t\right) .
\end{aligned}
$$

For short times

$$
\bar{\Theta}(t) \approx \frac{3 p_{0}}{2 a_{l} r_{0} \rho} t \text { and } \overline{\Delta r}(t) \approx \frac{p_{0}}{2 a_{l} \rho} t
$$

respectively, (cf. Fig. 1) so that $\bar{\Theta}$ and $\overline{\Delta r}$ increase linearly with $t$ although the individual modes set in proportional to $t^{2}$. Furthermore, we note that the function (9) has to be continued periodically when $t>2 r_{0} / a_{l}$. At the points where $t$ is a multiple of $2 r_{0} / a_{l}$ it's first derivative is discontinuous. Although we derived this formula only for short times, these discontinuities represent a 


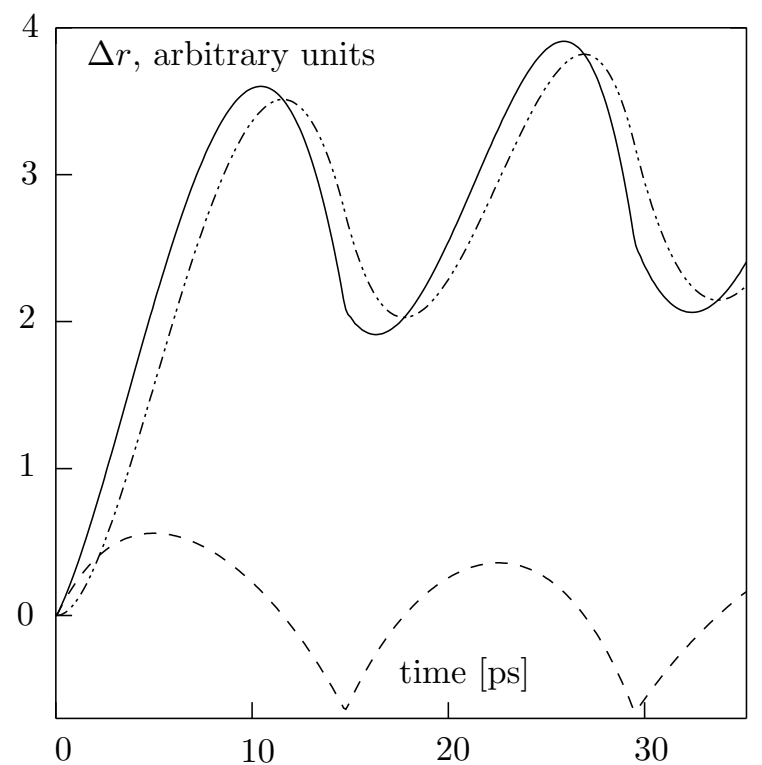

Figure 3: Electronic (dashed curve) and lattice (dash-dotted curve) contribution to the vibration (full curve) of a model gold cluster of radius $r_{0}=24.2 \mathrm{~nm}$. The electronic contribution is extremely anharmonic and determines the slope at $t=0$. The total vibration, and especially that part of it that is due to electronic pressure change, can be seen to have a discontinuous slope due to the sudden change of pressure.

general feature as they are due to the (in the limit considered here) infinitely rapid switching of the electronic pressure.

As the frequencies $\Omega_{n}$ are in general not multiples of each other, the general motion $\bar{\Theta}(t)$ will not be periodic. An exception occurs for liquids, where the asymptotic expression (9) becomes exact. In the latter case we can easily write down an analytic expression for the equilibrium shift: $\bar{\Theta}_{\mathrm{eq}}=p_{0} / 2 \rho a_{l}^{2}$. However, when deviations from the linear approximation eq. (11) become relevant, we shall have to take into account the pressure due to the hot lattice, too.

In ref. [4] detailed experimental data are presented for the case of gold nanoparticles with radius $r_{0}=24.2 \mathrm{~nm}$. These data where fitted to a model 
which takes only the fundamental breathing mode into account. The energy transfer from the electronic excitation into lattice excitations is described by a two temperature relaxation model. An even simpler approximation for the relaxation would be an exponential decay with a characteristic time $\tau_{\text {ep }}$. Neglecting again damping, the pressure can be modeled as

$$
p(t)=\frac{E}{V} \Theta(t)\left[\gamma_{\text {Fermi }} e^{-\frac{t}{\tau_{\mathrm{ep}}}}+\gamma_{\text {lattice }}\left(1-e^{-\frac{t}{\tau_{\mathrm{ep}}}}\right)\right] .
$$

For this time-dependence of pressure, the equation of motion (6) and (7), respectively, may be solved analytically using Laplace-transform techniques,

$$
\begin{aligned}
\frac{\Delta r(t)}{r_{0}}= & \frac{E}{V} \frac{1}{3 \rho a_{l}^{2}} \Theta(t) \sum_{n=1}^{\infty} I_{2, n}\left[\gamma_{\text {lattice }}\left(1-\cos \Omega_{n} t\right)\right. \\
& \left.+\left(\gamma_{\text {Fermi }}-\gamma_{\text {lattice }}\right) \frac{\Omega_{n}^{2} \tau_{\text {ep }}^{2}}{1+\Omega_{n}^{2} \tau_{\text {ep }}^{2}}\left(e^{-\frac{t}{\tau_{\mathrm{ep}}}}+\frac{\sin \Omega_{n} t}{\Omega_{n} \tau_{\text {ep }}}-\cos \Omega_{n} t\right)\right] .
\end{aligned}
$$

The solution with $\tau_{\text {ep }}=5$ ps is shown in Fig. 2. Besides the exact solution, which contains the contribution of all overtones, we also show the contribution of the fundamental mode. In the latter case, also the approximation $\frac{\left(K_{1} r_{0}\right)^{2}}{9} I_{2,1} \approx$ 1 was invoked, which can be seen to give rise to too large an amplitude of oscillation. We also show the fundamental mode when the electronic pressure is set to zero. This introduces a phase lag as found in refs. [3, 4]. However, the anharmonicity due to the overtones leads to a shift of the minima and maxima in comparison with the ones of the fundamental mode which is comparable to the shift due to the phase lag. For short times, the exact solution has a nonvanishing slope while the fundamental mode rises quadratically, as we already showed. In Fig. 3 we show the separate contributions of the lattice and electronic pressure, respectively. These curves are obtained by setting either $\gamma_{\text {Fermi }}$ or $\gamma_{\text {lattice }}$ equal to zero. It can be seen that the electronic pressure determines the 


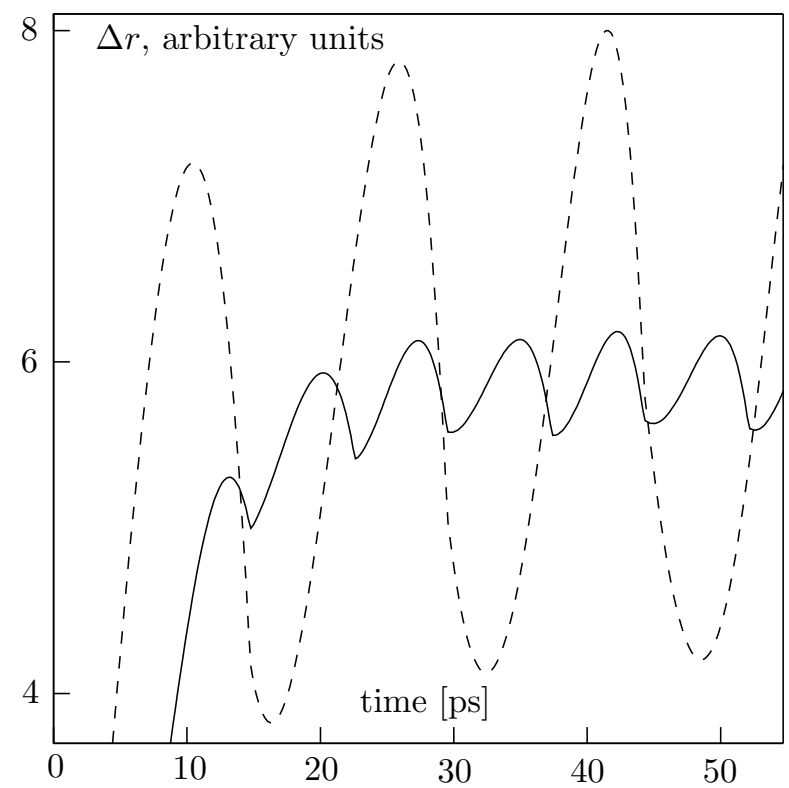

Figure 4: Simulation of the two pulse experiment [8]. The full curve describes the vibrational response due to a two pulse excitation with $p(t)=p_{0}[\Theta(t)+$ $\left.\Theta\left(t-\pi / \Omega_{1}\right)\right]$. For comparison, the dashed curve shows the response to a single pulse with $p(t)=2 p_{0} \Theta(t)$. It can be seen that the second pulse results in the stopping of the fundamental mode. The remaining oscillation is of higher frequency and lower amplitude as compared to the oscillation due to single pulse excitation. At the same time, it is much more anharmonic and the discontinuity of the slope is more pronounced. 
initial slope but dominates only for $t \ll \tau_{\text {ep }}$ and that the motion due to the electronic pressure is extremely anharmonic and has a discontinuous slope.

The function (13) describes the response of the system to optical excitation with an infinitely short laser pulse. Hence, it is the Greens function of the system and the response to more complex excitation sequences can be derived by convoluting this function with the time dependent laser intensity. Therefore, it is easy to simulate the outcome of the experiment described in ref. [8]: after exciting the nano-particle with a fs-laser pulse at $t=0$, a second laser pulse of equal intensity is applied at $t=\pi / \Omega_{1}$, which effectively stops the fundamental mode and leaves only the overtones. The radius change $\Delta r(t)+\Delta r\left(t-\pi / \Omega_{1}\right)$ with the $\Delta r$ given in eq. (13) is shown in Fig. 3. It can be seen that the oscillation is of lower amplitude and approximately double frequency as compared with the oscillation due to single pulse excitation. Furthermore, the oscillation is very anharmonic and the discontinuity of the slope is much more pronounced.

\section{Conclusion}

In this article the dynamics of vibrational modes in metal nanoparticles launched by an ultrashort laser pulse were considered. As new experiments show the importance of vibrational overtones and allow even to stop the fundamental mode, the methods presently used for the prediction of the vibrational dynamics were critically reviewed. It was found that these methods take only into account the fundamental vibrational mode and involved further approximations which tend to overestimate its importance.

In the second section, a set of ordinary linear differential equations (6) and (7) was derived from the fundamental equations of continuum mechanics which allow to calculate qualitatively the time depencence of the different vibrational modes once the time dependent electronic and lattice pressure is known. The 
decisive point in this analysis is the resolution of the pressure, which is approximately constant as a function of the coordinate $r$, in terms of the vibrational modes which are themselves proportional to spherical Bessel functions (4).

In the third section the theory developed is applied to model systems of different complexity. To obtain simple analytical formulas, instead of calculating the time dependence of the pressure using the two temperature model of ref. [4], a simple exponential decay of the electronic excitation into lattice excitation is assumed. Furthermore, the damping due to energy loss to the surrounding is neglected, as it is much slower than the timescales of the oscillations. Both assumptions are not essential to the argumentation.

In the first example, the short time vibrational response to a sudden change of pressure was analyzed. It was found that inclusion of the overtones leads to several qualitative differences with respect to models which neglect their influence: First, the radius increases linearly instead as quadratically for short times. Second, the position of the maxima and minima shifts due to anharmonicity. Third, the slope of the vibration becomes discontinuous. All these observations can be made also in the following more complex examples.

In the second example, the response (13) to a single ideally short laser pulse was simulated. The pressure (12) changes even after excitation due to the decay of the primary electronic excitation into lattice heat. As was to be expected, this slower change of the lattice pressure induces much less anharmonicities than the sudden change of the electronic pressure. The slope of the response to the electronic pressure is therefore much more discontinuous than the slope of the total response. The response function (13)may be used to construct the solution for an arbitrary excitation sequence.

In the third example the two pulse experiment described in ref. [8] was simulated. After excitation with a pulse at $t=0$, a second pulse is applied 
at $t=\pi / \Omega_{1}$ so that the fundamental mode induced by the second pulse is 180 degree out of phase with respect to the one by the first pulse. Hence the fundamental mode is stopped and only overtones remain. It was observed that the amplitude of the oscillation is reduced and the frequency approximately doubled, with the vibration being much more anharmonic as compared to the vibration induced by a single pulse.

\section{Acknowledgments}

The authors would like to thank M. A. El-Sayed for introduction into the subject matter.

\section{References}

[1] Del Fatti, N.; Tzortzakis, S.; Voisin, C.; Flytzanis, C.; Vallée, F. Physica B 1999, 263-264, 54-6.

[2] Hodak, J. H.; Henglein, A.; Hartland, G. V. J. Chem. Phys. 1999, 111, 8613-21.

[3] Perner, M.; Gresillon, S.; März, J.; von Plessen, G.; Feldmann, J.; Porstendorfer, J.; Berg, K.-J.; Berg, G. Phys. Rev. Lett. 2000, 85(4), 792-5.

[4] Hartland, G. V. J. Chem. Phys. 2002, 116(18), 8048-55.

[5] Huang, W.; Qian, W.; El-Sayed, M. A. Nano Lett. 2004, 4(9), 1741-7.

[6] Hartland, G. V. Annu. Rev. Phys. Chem. 2006, 57, 403-30.

[7] Nelet, A.; Crut, A.; Arbouet, A.; Del Fatti, N.; Vallée, F.; Portalès, H.; Saviot, L.; Duval, E. Appl. Surf. Sci. 2004, 226, 209-15.

[8] Arbouet, A.; Del Fatti, N.; Vallée, F. J. Chem. Phys. 2006, 124, 144701. 
[9] Sönnichsen, C.; Franzl, T.; Wilk, T.; von Plessen, G.; Feldmann, J. New J. Phys. 2002, 4, 93.1-93.8.

[10] Sommerfeld, A. Mechanik der deformierbaren Medien, Vol. II of Vorlesungen über Theoretische Physik; Verlag Harri Deutsch: Thun, 6. ed., 1992.

[11] Lamb, H. Proc. London Math. Soc. 1882, 13, 189.

[12] Dubrovskiy, V. A.; Morochnik, V. S. Earth Phys. 1981, 17, 494. 